\title{
Mean field and full field modelling of microstructure evolution and phase transformations during hot forming and cooling of low carbon steels
}

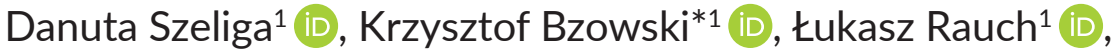 \\ Roman Kuziak ${ }^{2}$ iD, Maciej Pietrzyk ${ }^{1}$ (D)
}

${ }^{1}$ AGH University of Science and Technology, al. Mickiewicza 30, 30-059 Krakow, Poland.

${ }^{2}$ Łukasiewicz Research Network, Institute for Ferrous Metallurgy, ul. K. Miarki 12, 44-100 Gliwice, Poland.

\begin{abstract}
The paper describes a critical comparison of mean field and full field approaches to modelling hot deformation/controlled cooling sequences for steels. Classification of the models, based on the balance between predictive capabilities and computing costs, is presented. Mean field models, which describe microstructure evolution and phase transformations were connected with thermomechanical finite element program and applied to simulation of the hot strip rolling process and cooling of tubes after hot rolling. Full field model described in the paper is a connection of the finite element (FE) and level set (LSM) methods. These methods were used to simulate heating/cooling sequence in the continuous annealing line. A suggestion to use a stochastic model as a bridge between mean field and full field approaches is made.
\end{abstract}

Keywords: Mean field models, full field models, finite element method, level set method.

\section{Introduction}

Metal alloys, including steels, are composed of numerous grains, each grain having its own crystallographic orientation describing the atoms arrangement in space. The grain size for commercial construction steels is typically between 10 and $100 \mu \mathrm{m}$ and the grains are separated by low or high angle boundaries. Beyond the chemical composition, the microstructure features represented by size, shape and orientation of grains influence the properties of steels. To obtain an intermediate steel product, the cast metal alloy is hot worked and cooled in dedicated devices like laminar cooling for strips (Liu et al., 2012) or TempCore sys- tem for rods (Nino, 2007). Design of the manufacturing technology requires models, which can predict mentioned above microstructure features with reasonable accuracy.

A variety of microstructure evolution and phase transformation models have been developed to date. Mean field and full field material models are distinguished in the scientific literature. In the former, the microstructure is implicitly represented by closed form equations describing microstructure evolution including grain size, dislocation density (uniform per grain), kinetics of phase transformations and as a result, volume fraction of structural components, etc. The latter are based on an explicit microstructure representation

*Corresponding author: kbzowski@agh.edu.pl

ORCID ID's: 0000-0002-2915-8317 (D. Szeliga), 0000-0002-1784-2584 (K. Bzowski), 0000-0001-5366-743X (Ł. Rauch), 0000-0003-4965-5123 (R. Kuziak), 0000-0002-1473-4625 (M. Pietrzyk)

(c) 2020 Authors. This is an open access publication, which can be used, distributed and reproduced in any medium according to the Creative Commons CC-BY 4.0 License requiring that the original work has been properly cited. 
using Representative Volume Element (RVE). The present work is focused on opposing mean field models of microstructure evolution and phase transformations to a selected full field model based on coupling a finite element solution of the diffusion equation with a level set method.

One has to realize that calculations of microstructure evolution at the micro scale require information about strains and temperatures in the macro scale during processing. It means that microstructure evolution models, which are local in nature, have to be connected with the macroscale model and a multiscale methodology (Allix, 2006) has to be applied. The most common multiscale approach is a connection of the thermomechanical finite element (FE) model in the macro scale with micro scale models. To obtain a full and reliable picture of the microstructure in the whole product, the micro scale model should be solved in each Gauss point of the FE mesh. It means that computing costs are crucial for the practical application of this approach. It is obvious that predictive capabilities of the full field models are much wider but they involve much larger computing costs. A thorough review of various material models and discussion of their predictive capabilities versus computing costs can be found in Pietrzyk et al. (2015). Based on this review, a classification of the models shown in Figure 1 was proposed.

Information about all of the models in Figure 1 is given in Chapter 3 of this paper. It can be seen from Figure 1 that selection of the proper model for a specific application is a challenge. Therefore, the main objective of the present work was a review of the material modelling methods in distinction into mean field and full field approaches. Following this, a proposition of the extension of the internal variable mean field modelling by an introduction of the stochastic variables is described. This new model should be located in the place marked with a star in Figure 1.

\section{Methodology}

All of the models discussed in this paper were developed by the authors and have been described in earlier publications, with a relevant reference given for each model. The present work is focused on critical evaluation of these models and discussion of their efficiency. Following this selected results of case studies for both groups of the models are presented. The case studies involve new applications of the models for the simulation of industrial metallurgical processes. Since our intention was to address practical applications of the models, the full field model is limited to the phase transformations during which microstructure and properties of the final product are determined. The simulation of hot forming is constrained to mean field models. We assumed that input data for the phase transformation models (grain size, dislocation density) can be averaged.

The numerical tests showed motivation for searching for a more advanced mean field model, which will have extended predictive capabilities combined with only slight increase of the computing costs.

\section{State of the art in materials modelling}

\subsection{Mean field models}

A need for the numerical modelling of metal forming including microstructure evolution was noticed more than thirty years ago (Pietrzyk, 1990; Karhausen \& Kopp, 1992). Historically, modelling was focused on the prediction of strains, stresses and temperatures in the macro scale using FE models, which were coupled with the mean field models describing microstructure evolution and fully coupled thermal-mechanical-micro-

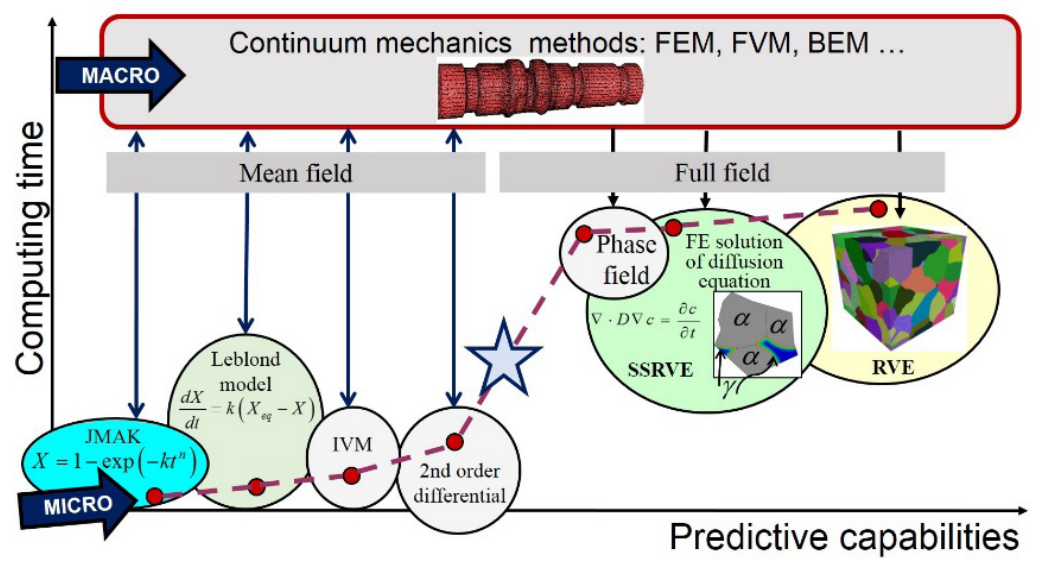

Fig. 1. Classification of the material models in the coordinate system: predictive capabilities vs. computing costs 
structural simulations became possible. The modelling of recrystallization and phase transformations kinetics has been for a long time based on JMAK (JohnsonMehl-Avrami-Kolmogorov) equation (Колмогоров, 1937; Avrami, 1939; Johnson \& Mehl, 1939):

$$
X=1-\exp \left(-k t^{n}\right)
$$

where: $X-$ transformed volume fraction, $t$ - time, $k, n$ - coefficients.

JMAK equation was developed long before the multiscale modelling was proposed. A review of the development of metal forming modelling accounting for the microstructure evolution can be found in Pietrzyk and Madej (2017). The JMAK equation was originally developed for isothermal conditions, therefore, Scheil additivity rule (Scheil, 1935) had to be applied to account for changes of the temperature. To avoid this, Leblond and Devaux (1984) proposed differential equation, in which the rate of transformation is proportional to the distance from the equilibrium:

$$
\frac{d X}{d t}=k\left(X_{e q}-X\right)
$$

where: $X_{e q}$ - equilibrium volume fractions of a new phase in the current temperature, $k$ - coefficient.

Several upgrades of the JMAK equation are described in the literature (Pietrzyk et al., 2015). They include:

- According to Pietrzyk and Kuziak (2012) coefficient $k$ is temperature dependent. Various functions are used to describe this relation but modified Gauss function proposed by Donnay et al. (1996) is the most common because it allows to reproduce $\mathrm{C}$ shape curve characteristic for the isothermal transformations.

- Calculations of carbon concentration in the austenite during both ferrite and bainite transformations were added. Changes of the carbon concentration are calculated from the mass balance (Pietrzyk \& Kuziak, 2012).

- The $T_{0}$ temperature concept for bainitic transformation proposed by Bhadeshia and Honeycombe (2006) was added. The carbon concentration calculated from the $T_{0}$ line is used in the model to control the progress of the bainite transformation.

- Control of the carbon concentration in the austenite during ferrite and bainite transformations allow the prediction of the occurrence of the retained austenite when the martensite start temperature drops below room temperature.
Both equations (1) and (2) require the introduction of the incubation time to account for the delay caused by the nucleation stage. To avoid this an upgrade of equation (2) was proposed by Milenin et al. (2015). Second order differential equation was proposed to describe kinetics of the growth of the new phase:

$$
B_{1}^{2} \frac{d^{2} X}{d t^{2}}+B_{2} \frac{d X}{d t}+X=X_{e q}
$$

where $B_{1}, B_{2}$ - time constants defined as functions of the temperature

Equation (3) describes second order inertia term. Time constant $B_{1}$ represents delay of the response and accounts for the nucleation process. Time constant $B_{2}$ is responsible for the growth of the ferrite phase, so it was correlated with the mobility of the interface and diffusion coefficient.

All of the mean field models discussed above use external variables as independent ones. This approach does not allow them to account for the history of deformation. When the conditions of the process change rapidly, the response of the model moves to a new equation of state without any transient state. In metallic materials a delay is observed (Urcola \& Sellars, 1987) and it is due to the inertia of the microstructural phenomena. Therefore, models which use internal variables (IVM) were developed to eliminate this disadvantage. Dislocation density is the internal variable, which is commonly used for metallic materials. Models describing evolution of dislocations populations are extensively used to simulate hot forming processes. The evolution equation is based on the fundamental works of Kocks, Estrin and Mecking (KEM model) (Mecking \& Kocks, 1981; Estrin \& Mecking, 1984), with recrystallization term added:

$$
\frac{d \rho(t)}{d t}=A_{1} \dot{\varepsilon}-A_{2} \rho \dot{\varepsilon}-A_{3} \rho^{a_{8}} \cdot \mathbf{1}_{\left(t_{c r},+\infty\right)}(t) \cdot \rho\left(t-t_{c r}\right)
$$

where: $\rho$ - dislocation density, $t$ - time, $\dot{\varepsilon}$-strain rate, $t_{c r}$ - time at which critical dislocation density for recrystallization is reached, $\mathbf{1}_{\left(t_{c r},+\infty\right)}(t)$ - indicator function, $A_{1}, A_{2}, A_{3}$ - coefficients.

The developed models were verified by means of a comparison of the predictions with the results of dilatometric tests (Bzowski et al., 2017; Kuziak et al., 2018) and the good predictive capability of the models was confirmed. The described mean field models, when implemented in the FE program, describe microstructure evolution during processes of hot forming and cooling. The connection of the FE macro model with mean field micro model does not cause an increase of the computing costs. Selected examples of such simulations are presented in section 4.1. 


\subsection{Full field models}

The modern modelling of materials during processing is based on the observation that the measured material response depends on microstructural features such as the grain size, the texture, the porosity etc. As it is seen in Figure 1, the mean field models are very fast but their predictive capabilities are limited and they are not able to reproduce mentioned above microstructural features. Therefore, simulations at a polycrystalline scale, called full field simulations, were proposed in the last decades to predict local evolution phenomena in the microstructure. The idea of the Representative Volume Element (RVE) is used to represent microstructure explicitly.

In practical applications to industrial processes, material models have to be coupled with macro models, which calculate stress, strain and temperature history. It means that multiscale approach has to be applied. The majority of developed multi-scale models can be classified into two groups: upscaling and concurrent multi-scale computing techniques (Allix, 2006); see also authors' publications (Pietrzyk \& Madej, 2017; Madej et al., 2008). The major difference in the two approaches lies in the concept of physical area description at various length scales. In the concurrent models, the same physical area is discretized with different techniques capable of capturing phenomena operating at a particular length scale. The upscaling approach is directly related to a concept that was already intuitively used by researchers in the 1990's (Pietrzyk, 1990). In today solutions, the lower length scale models are attached to the upper length scale in the form of the mentioned earlier RVEs (Bargmann et al., 2018). To describe the macro scale computational domain, the classical finite element method is usually used, but solutions with finite volume method or boundary element method or meshless methods can be encountered in the literature.

When full field micro scale material models are required, various continuum or discrete methods can be used. Evolution of the microstructure including phase transformations can be simulated using such methods as phase field (PF), level set (LS), Monte Carlo (MC) or Cellular Automata (CA); see review of these methods presented by Madej (2017).

CA seem to be the most commonly used in modelling of phase transformations. First applications of the CA to modelling microstructure evolution appeared at the end of the 20th century and were mainly dedicated to recrystallization (Marx et al., 1999). Many papers were published at the beginning of the $21^{\text {st }}$ century. The focus was still on modelling recrystallization (Raabe, 2002) and grain growth (Raghavan \& Sahay,
2007) but several papers dedicated to phase transformation were published as well (Lan et al., 2004). The authors' CA model, describing the transformation of ferritic-pearlitic microstructure into austenite during heating, is discussed in Halder et al. (2014). Extensive research on the applications of CA to modelling microstructure evolution is continued (Song et al., 2015) and recent focus is on $3 \mathrm{D}$ solution and reduction of computing costs by distributed computing; see eg. Lian et al. (2018), and on application to different metallic materials as well as experimental validation (Contieri et al., 2017).

Publication of Abinandanan et al. (1998) can be considered the first application of the Monte Carlo method dedicated directly to diffusional phase transformations. Clouet \& Soisson (2010) applied the atomistic diffusion model and $\mathrm{MC}$ algorithm to model solid state transformations.

Phase field (PF) and Level Set (LS) have emerged as the most powerful methods for modelling many types of microstructure evolution processes which involve moving interfaces. The latter is discussed in the section 4.2, where the authors' model is presented. Detailed description of the PF method was presented by Mecozzi et al. (2008). The PF model treats a polycrystalline system in an integral manner. A set of continuous phase-field variables (space-time functions) representing individual grains are defined. These variables have a constant value inside the grains and change continuously over a diffuse boundary. It is the main advantage of the PF method, which transfers discontinuous interface into a gradual change of the two corresponding phase field variables. Among many published PF papers those dedicated to phase transformations should be mentioned (Rudnizki et al., 2011; Steinbach \& Apel, 2006). Recently published research focus on the $3 D$ solution (Bollada et al., 2015) and multi PF approach (see Mecozzi et al., 2016; Liu et al., 2018). Good review of the FP models can be also found in the book by Choudhury (2013).

A review of the full field models of diffusional phase transformations including CA, MC and MD methods is presented by Liu et al. (2018). A thorough comparative discussion of the mean field and full field approaches to modelling dynamic recrystallization is presented in the Maire's PhD thesis (2018). A similar discussion regarding modelling of grain growth can be found in Furstoss et al. (2020) while publication by Pietrzyk and Madej (2017) presents a general comparison of the mean field and full field material models in describing microstructure evolution during hot forming and controlled cooling of metallic materials. 


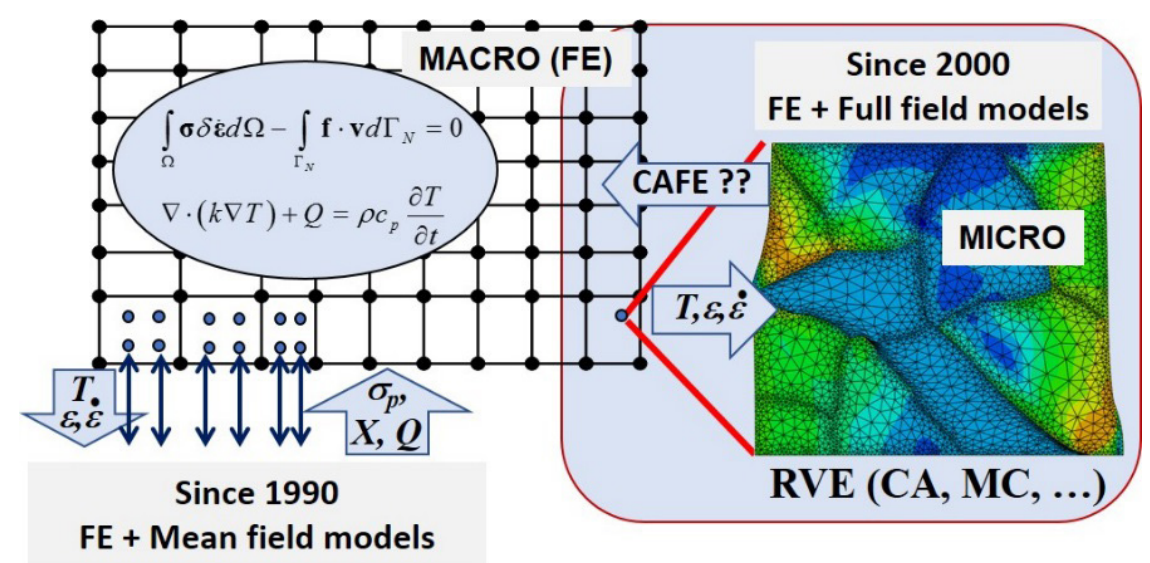

Fig. 2. Schematic illustration of the multiscale approaches based on mean field and full field micro scale models

There are fewer papers on the application of molecular dynamics (MD) to modelling microstructure evolution (Le et al., 2019) and they are dedicated special alloys or nanostructures, a method not discussed in the present paper.

FE method can be also used to describe phenomena in the micro scale and this approach is referred to as $\mathrm{FE}^{2}$ method (Schröder, 2014). Moreover, Level Set (Bzowski, 2019; Fausty et al., 2020) and Phase Field or MultiPF can be implemented in the FE framework and these solutions are also classified as $\mathrm{FE}^{2}$.

All the methods which are described above are allocated under the full field models that can take into account microstructure morphology in an explicit manner. Enormous progress in multiscale modelling concepts and development of sophisticated metallic material models during last two decades has been documented in series of books (see, for example Janssens et al., 2007; Fish, 2009, 2013). The micro scale modelling approach developed by the authors is described in Chapter 4.

In the case of multiscale approach with mean field micro scale models, upper length scale constitutive material description is constructed by the outcome from lower length scale models. It means that feedback between upper scale and lower scale is introduced (Fig. 1). Information about strains, strain rates, stresses and temperatures is sent to micro scale and used in material models. The micro scale models return to the macro scale such parameters as flow stress, recrystallized volume fraction, phase fraction and heat generated due to recalescence. This approach is known as full coupling of solution fields. Due to the very high computing costs, such feedback is rarely used for the full field material models, although examples of complete coupling solutions can be found in the literature, see for example publications on the CAFE method (Cellular Automate Finite Element) (Das et al., 2002).
Schematic illustration of the multiscale approaches based on mean field and full field micro scale models is shown in Figure 2.

\section{Case studies}

\subsection{Mean field}

The 2D steady state FE solution, which was used in the macro scale calculations, is based on the rigid-plastic thermo-mechanical finite element approach proposed by Kobayashi et al. (1989). A detailed description of the algorithm and the program, which was used in the present work, is given by Pietrzyk (2000). A simple mesh with 9 elements through the half thickness (symmetry) and 20 elements along the roll gap was satisfactory to obtain good agreement with experimental data (Bzowski et al., 2017). In consequence, the model was very fast and could be used in the optimization loop. All rolling parameters are described by Bzowski et al. (2017), where the computer system VirtRoll is presented. FE coupled with mean field microstructure evolution model was used to simulate and optimize various manufacturing processes based on hot metal forming followed by controlled cooling.

Two examples of simulations are presented below. The first is hot strip rolling followed by laminar cooling of Dual Phase (DP) steel strips. All mean field material models for the considered DP steel (S406) are given by Bzowski et al. (2017). Rolling of the $3 \mathrm{~mm}$ thick strip was considered. Temperatures in the 6 stand finishing train calculated by the FE code are shown in Figure 3a and rolling forces as well as austenite grain sizes are shown in Figure 3b. Slab thickness at the entry to the finishing train of the strip mill was $60 \mathrm{~mm}$. Microstructure evolution, including recrystallization and grain 
growth, was calculated by the mean field model, which was solved in each Gauss point of the FE mesh. Grain growth was calculated globally for the whole interpass time, therefore, it is represented by linear relation in Figure $3 b$.

The same approach was used to optimize the process of laminar cooling after hot strip rolling. The JMAK equation was used as micro scale model of phase transformations and was coupled with FE simulations of temperature changes in the macro scale. This multiscale model was a basis of the computer system VirtRoll, which allows to design optimal parameters of the hot strip rolling for steels. This system is described by Bzowski et al. (2017). The objective of the optimization of the laminar cooling, which was performed in the present work, was to obtain $25 \%$ of martensite in the microstructure without pearlite and bainite. The results for the two sets of rolling parameters are presented below: A) $h_{6}=3 \mathrm{~mm} ; v_{6}=7.5 \mathrm{~m} / \mathrm{s}$, B) $h_{6}=3 \mathrm{~mm}$; $v_{6}=6 \mathrm{~m} / \mathrm{s}$, where $h_{6}$ and $v_{6}$ are strip thickness and rolling velocity in the last stand, respectively. Optimal temperature cycles, which give $25 \%$ of the martensite, are shown in Figure 4a. Kinetics of ferrite and martensite transformations for these temperatures is shown in Figure $4 \mathrm{~b}$. It is seen that $25 \%$ of martensite was predicted in both cases.

a)

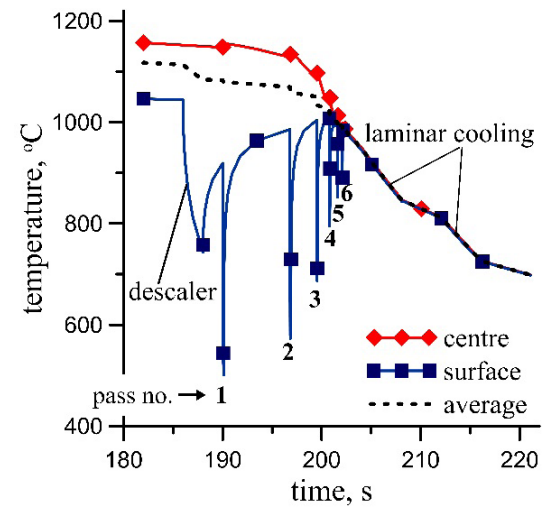

b)

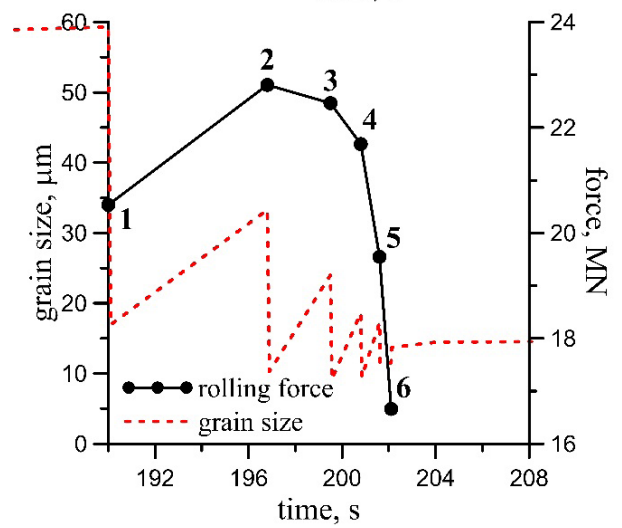

Fig. 3. Temperatures (a), rolling forces and grain size (b) in the finishing train calculated by the FE code coupled with the mean field microstructure evolution model a)

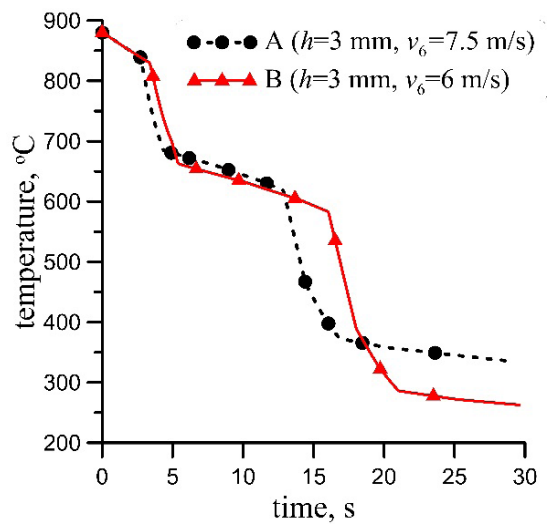

b)

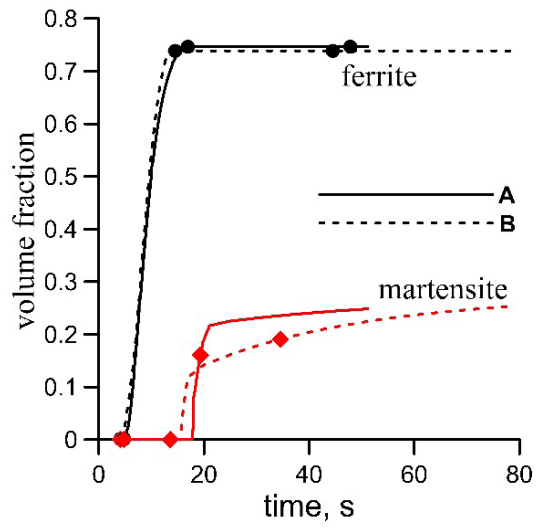

Fig. 4. Optimal temperatures, which give $25 \%$ of the martensite (a) and kinetics of ferrite and martensite transformations for these temperatures (b)

Alternatively an upgrade of the Leblond model was used in this simulation. The results were similar and the computing times were the same. Including micro scale simulations did not influence the macro scale computing time for either the JMAK or Leblond models.

Similarly, the IVM model based on equation (4) was implemented in the FE code for hot strip rolling and simulation of the distribution of the dislocation density in the roll gap became possible. Full coupling was applied, what means that flow stress calculated by the IVM model was returned to the constitutive law in the FE model. The results of these simulations are presented by Morkisz et al. (2019) and are not repeated here. Explicit finite difference solution of equation (4) is performed in each time step of the FE method and, in consequence, this approach does not influence the computing costs of the FE code.

The second example considered in the present work was the cooling of the low carbon steel tubes. This process is described by Kuziak et al. (2018). The chemical composition and the phase transformation model of the investigated steel are given in this publication, as well. Three cooling strategies of tubes were simulated:

- cooling in the free air,

- cooling in the air under pressure,

- cooling in the water mist. 
The objective was to compare the kinetics of transformation for different cooling schedules. The tube measuring $32 \times 4.2 \mathrm{~mm}$ was considered and the selected results are presented in Figure 5. These results are for the inner surface of the tube but variations of phase volume fractions through the wall thickness are small. Figure 5a shows kinetics of transformation for the three cooling methods. Ferrite and bainite are the only structural components. The volume fraction of bainite increases when cooling rate increases. Since FE code was used in the macro scale, the coupled model enabled prediction of the microstructure at the cross section of the tube. It is particularly useful when asymmetrical cooling is applied. Figure $5 \mathrm{~b}$ shows the calculated distribution of the bainite volume fraction at the tube cross section after the cooling sequence, in which water mist was applied from the right side of the tube only. The tube measuring $38 \times 5.6 \mathrm{~mm}$ was considered in this case. The asymmetrical distribution of the phase composition is seen in this figure.

a)

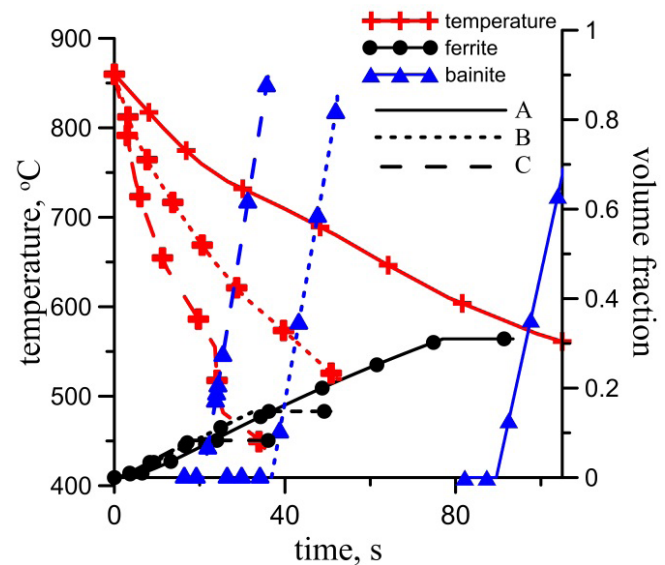

b)

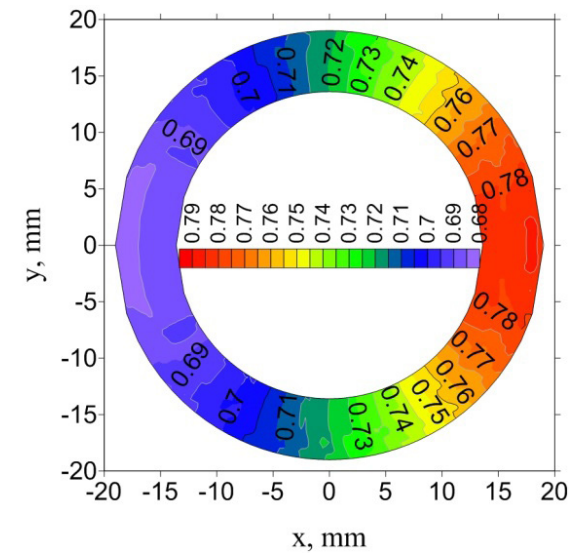

Fig. 5. Kinetics of transformation for the three cooling methods of the tube (a) and distribution of the bainite volume fraction at the cross section of the tube after asymmetrical cooling sequence (b)

Selected results of simulations using FE code in the micro scale combined with the mean field material models in the micro scale were presented in this section. Several similar simulations for various metal forming processes were performed by the authors. All these numerical tests allow to conclude that computing times are very short. For example, simulation of the hot strip mill composed of 12 passes took less than one minute. Simulations of cooling of tubes were even shorter. In both cases the calculations were handled single threaded on the typical desktop computer. The time step was adjusted adaptively depending on the convergence of the solution. On the other hand, the predictive capabilities of this approach are limited to average values of the microstructural parameters, average grain size, average dislocation density and average phase composition. Application of the RVE in the micro scale is an alternative which is discussed in the next section.

\subsection{Full field}

Among several methods which can be used to simulate microstructure evolution in the micro scale (CA, MC, MD, FE, LSM), a combination of the FE and LSM (Leve Set Method) was applied in the example presented in this section. The solution was performed in the Statistically Similar Representative Volume Element (SSRVE), which is a statistical simplification of the RVE (Schröder et al., 2011). Details of the FE + LSM solution are given by Bzowski et al. (2018) and in the PhD thesis (Bzowski, 2019).

Whatever the modelling method used, it has to reproduce properly metallurgical phenomena behind the phase transformations. Due to limited space the metallurgical background cannot be discussed in detail. The present model is based, in general, on thermodynamic aspects of nucleation and growth described well in the book by Christian (1975). Modelling aspects for these phenomena are discussed well in the book by Pereloma and Edmonds (2012), in which a summary of the current state of research on phase transformations in steels and its implications for the emergence of new steels with enhanced engineering properties is presented with the focus on the diffusion-controlled phase transformations.

Nucleation of a new phase is based on the theory described by Strangwood (2012), which distinguishes initial heterogeneous nucleation and site saturation and accounts for the grain size of the parent phase; see Bzowski (2019) for details. Growth is simulated using LSM approach. LSM is particularly useful when the interface motion controls the investigated phenomenon and reliable modelling of this motion is crucial. The interfaces can be represented numerically by either explicit or implicit methods. The front tracking or cellular 
automata approaches belong to the first group. In these methods, the grain boundaries are tracked by piecewise linear or curved segments or cells. Front tracking method requires special treatment of triple junctions as well as handling topological changes (splitting and merging). Implicit grain representation eliminates presented difficulties. LSM developed by Osher and Sethian (1988) is one of the most popular implicit interface representations and evolution procedure. A major advantage of the LSM is natural merging and splitting regions without grid modifications when the interface moves. The time consuming remeshing stage is not needed. Moreover, no additional markers or tracking methods are required to evaluate interface. The level set function divides the domain into two regions, distinguished by function sign (positive and negative). The zero level set acts as a marker that determines the position of the interface. Application of the LSM to describe microstructure evolution are frequent. Bernacki et al. (2008) presented application of the LS method to simulation of static recrystallization, Håkan (2013) used this method to modelling of dynamic recrystallization. Regarding the LS grain growth modelling, it was addressed in numerous papers (eg., Bernacki et al., 2008) applied LS for the modelling of topological evolution of the grain structure during recrystallization and the most recent paper (Fausty et al., 2020) is focus on modelling anisotropic grain growth.

In the present model, the LS method was applied to simulate the transformation of the pearlite/ferrite microstructure into austenite during heating and ferritic transformation during cooling in steels. Briefly, the phase interface is represented numerically by the level set function, which divides the domain into two regions distinguished by the sign of the function $\phi$ (positive and negative). The zero level set acts as a marker that determines the position of the interface. The function $\phi$ represents the distance between considered point and the interface. The general idea is to move zero level set $(\Gamma)$ with predefined velocity $v$ by solving equation of motion. Velocity depends on internal or external forces and the equation of motion governing $\phi$ is:

$$
\frac{\partial \phi}{\partial t}+F|\nabla \phi|=0
$$

where: $F$ - a vector function defined at each degree of freedom.

In principle, velocity can only be reliably calculated on the interface. The velocity extension algorithm is applied, which involves assigning to each degree of freedom in the mesh a velocity vector from the near- est point on the closest interface. The definition of interface velocity evaluation is described by Fang et al. (2018), capillarity term is neglected as of second order far from the triple junctions:

$$
F=M \Delta G
$$

where: $M-$ mobility of the interface, $\Delta G$ - driving force of transformation given by:

$$
\Delta G=\chi\left(C_{\mathrm{eq}}^{\gamma \alpha}-C^{\gamma}\right)
$$

where: $\chi-$ proportionality factor, $C_{\mathrm{eq}}^{\gamma \alpha}-$ equilibrium carbon concentration in austenite, $C_{\gamma}$ - average carbon concentration in austenite.

The solution based on the LSM is split into four basic stages: initialization, determination of the velocity field, interface evolution and reinitialization. In the initialization phase, base position of the interface is defined by assigned value of the signed distance function $\phi$ for all degrees of freedom. Interface translation is done by solving the motion equation (5) using FE method. The procedure is described in detail by Roh and Kikuchi (2002). LSM is applicable to problems where distinction between the two regions is needed. This method was coupled with the FE solution of the carbon diffusion equation:

$$
\frac{\partial c}{\partial t}=\nabla \cdot D \nabla c
$$

where: $D$ - diffusion coefficient, $c$ - carbon concentration.

Within austenite, the diffusion coefficient is constant at each time step. Globally, its magnitude depends on the current temperature applied at a given time step. In the case of ferrite grains, the diffusion of elements is not accounted for. All mesh elements belonging to ferrite have zero degrees of freedom and the diffusion equation is not solved on them.

Steel containing $0.11 \% \mathrm{C}, 0.19 \% \mathrm{Si}, 1.45 \% \mathrm{Mn}$ and small additions of $\mathrm{V}, \mathrm{Nb}$ and $\mathrm{Ti}$ was used as an example for a numerical test. A typical continuous annealing process for strips was recreated to demonstrate capabilities of the model. Input as ferrite-pearlite microstructure image was obtained after the cold rolling of steel sheet. Based on the picture of the microstructure, a mesh grid was generated. Constant heating rate $\left(3^{\circ} \mathrm{C} / \mathrm{s}\right)$ up to $900^{\circ} \mathrm{C}$ followed by cooling to $420^{\circ} \mathrm{C}$ with constant cooling rate of $10^{\circ} \mathrm{C} / \mathrm{s}$ were applied as boundary conditions. Typical results showing carbon distribution as well as microstructure morphology after heating and cooling stages are presented in Figure 6. No uniform carbon concentration is observed in the austenite. 
a)

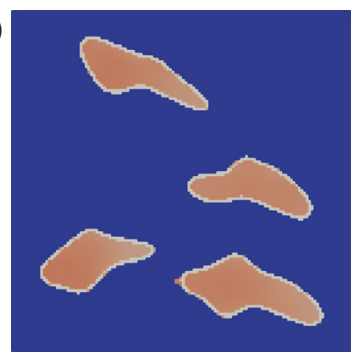

c)
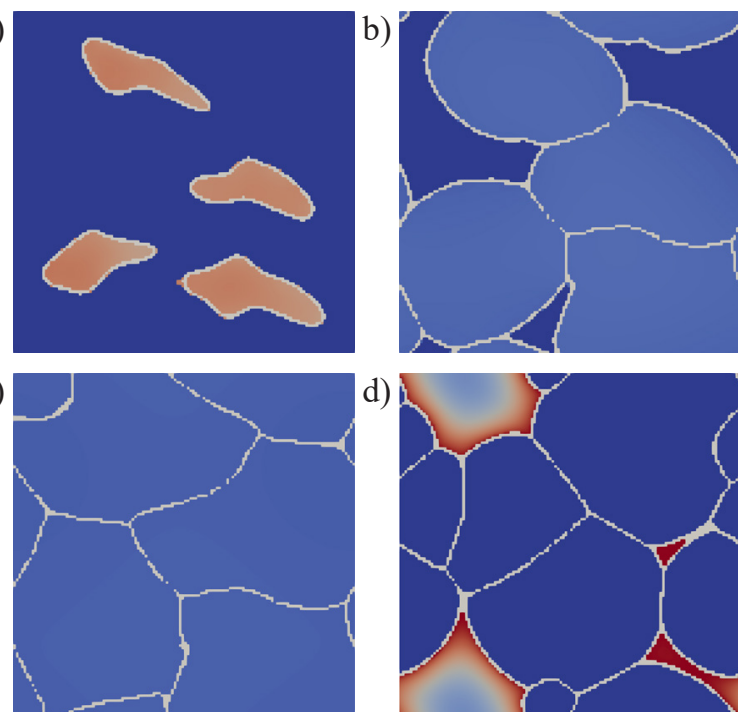

d)

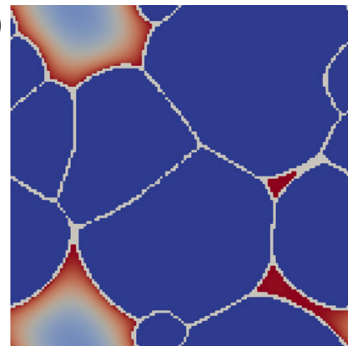

Concentration

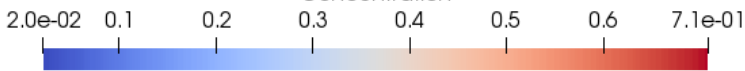

Fig. 6. Input ferrite-perlite microstructure (a), austenite microstructure at $830^{\circ} \mathrm{C}$ (b), austenite microstructure at $900^{\circ} \mathrm{C}$ (c) and ferrite-austenite microstructure with carbon concentration distribution in the austenite at $420^{\circ} \mathrm{C}$ (d).

The presented results show the extensive predictive capabilities of the full field model, which can predict the morphology of the microstructure and distribution of the carbon concentration in the austenite. The model can be easily extended by including the diffusion of other elements in the steel. On the other hand, the computing costs of this model are high. The results in Figure 6 concern only one point in the material. The computing time for the continuous annealing sequence was 50 minutes on a typical quadcore desktop computer. Attaching this model to each Gauss point of the FE mesh in the macro scale would lead to unacceptable computing times.

\section{Conclusions, discussion and motivation for future work}

Numerical tests allowed us to compare the models describing hot deformation and controlled cooling of steel products and the following conclusions were drawn:

- Mean field models are fast and they do not influence computing time of the macro scale FE simulation. These models can be solved at each Gauss integration point of the FE mesh.

- Both JMAK and upgrade Leblond models give similar results and they can be used alternatively. Since the latter model is based on a differential equation with respect to time it is performs better for the processes with varying temperatures. It does not need the application of the additivity rule. On the other hand, the JMAK model has been extensively researched and many upgrades of this model are described in the literature.

- Full field models have extensive predictive capabilities, which include morphology of the microstructure and segregation of the chemical composition. The computing costs of this model are high and attaching this model to each Gauss point of the FE mesh in the macro scale leads to unacceptable computing times. Thus, in practical applications, the solution is performed in only a few selected points of the product.

Since full field models are generally greedy in terms of computational costs, phenomenological laws can be used as an alternative to predict mean quantities such as recrystallized fractions, average dislocation density, mean grain sizes or average phase composition. If mean field models can be attached to each Gauss point in the FE mesh without a noticeable increase in computing costs, such a solution is practically impossible for the full field models. But the mean field models are often based on many simplifying assumptions. Therefore, a search for the extension of the predictive capabilities of the mean field models is needed. It seems that the solution of the algebraic equations describing microstructure evolution for the stochastic variables should supply additional information about the distribution of the microstructural features, while the computing costs should increase only slightly. The IVM model based on equation (4) was selected for such a solution. The main weak point of equation (4) is the critical time $t_{c r}$ introduced to predict dynamic recrystallization, which is not physical. In a real material, the recrystallization phenomenon occurs in various parts at different times. Therefore, to avoid this "artificial" time and to build a model consistent with physics, the stochastic model should be considered. Accounting for the stochastic character of the evolution of the dislocation density and discretization in time transforms equation (4) as follows:

$$
\begin{gathered}
\rho\left(t_{i}\right)=\rho\left(t_{0}\right)\left[1-\xi\left(t_{i-1}\right)\right]+ \\
\left\{\rho\left(t_{i-1}\right)+\left[A_{1} \dot{\varepsilon}-A_{2} \rho\left(t_{i-1}\right) \dot{\varepsilon}\right] \Delta t\right\} \xi\left(t_{i-1}\right)
\end{gathered}
$$

Parameter $\xi\left(t_{i}\right)$ is a stochastic variable, such that:

$$
\begin{gathered}
\mathbf{P}\left[\xi\left(t_{i}\right)=0\right]=\left\{\begin{array}{c}
p \text { if } p<\Delta t \\
\Delta t \text { otherwise }
\end{array}\right. \\
\mathbf{P}\left[\xi\left(t_{i}\right)=1\right]=1-\mathbf{P}\left[\xi\left(t_{i}\right)=0\right]
\end{gathered}
$$

where $p$ - probability that material recrystallizes at the current conditions of the process.

The mean field model based on the IVM with stochastic variable emerges as a compromise between full field models and analytical laws. This model will supply information about the heterogeneity of dislocation 
density and grain size, which will allow the evaluation of the local fracture resistance of the material.

An attempt to solve the dislocation evolution equation for the stochastic variable will be the subject of future work.

\section{Acknowledgements}

Financial assistance of the National Science Foundation in Poland (NCN), project no. 2017/25/B/ST8/01823, is acknowledged.

\section{References}

Abinandanan, T.A., Haider, F., \& Martin, G. (1998). Computer simulations of diffusional phase transformations: Monte Carlo algorithm and application to precipitation of ordered phases. Acta Materialia, 46(12), 4243-4255.

Allix, O. (2006). Multiscale Strategy for Solving Industrial Problems. In C.A. Motasoares, J.A.C. Martins, H.C. Rodrigues, J.A.C. Ambrósio, C.A.B. Pina, C.M. Motasoares, E.B.R. Pereira, J. Folgado (Eds.), III European Conference on Computational Mechanics. Solids, Structures and Coupled Problems in Engineering: Book of Abstracts (pp. 107-126). Springer.

Avrami, M. (1939). Kinetics of phase change. I. General theory. Journal of Chemical Physics, 7(12), 1103-1112.

Bargmann, S., Klusemann, B., Markmann, J., Schnabel, J.E., Schneider, K., Soyarslan, C., \& Wilmers, J. (2018). Generation of 3D representative volume elements for heterogeneous materials: A review. Progress in Materials Science, 96, 322-384.

Bernacki, M., Chastel, Y., Coupez, T., \& Logé, R.E. (2008). Level set framework for the numerical modelling of primary recrystallization in polycrystalline materials. Scripta Materialia, 58(12), 1129-1132.

Bhadeshia, H.K.D.H., \& Honeycombe, R.W.K. (2006). Steels. Microstructure and properties (3 ${ }^{\text {rd }}$ ed.). Elsevier, Butterworth-Heinemann.

Bollada, P.C., Goodyer, C.E., Jimack, P.K., Mullis, A.M., \& Yang, F.W. (2015). Three dimensional thermal-solute phase field simulation of binary alloy solidification. Journal of Computational Physics, 287, 130-150.

Bzowski, K. (2019). Wykorzystanie statystycznej reprezentacji mikrostruktury do modelowania przemian fazowych w stalach DP poprzez rozwiązanie równania dyfuzji ( $\mathrm{PhD}$ thesis). Akademia Górniczo-Hutnicza, Kraków.

Bzowski, K., Kitowski, J., Kuziak, R., Uranga, P., Gutierrez, I., Jacolot, R., Rauch, Ł., \& Pietrzyk, M. (2017). Development of the material database for the VirtRoll computer system dedicated to design of an optimal hot strip rolling technology. Computer Methods in Materials Science, 17(4), 225-246.

Bzowski, K., Rauch, Ł., \& Pietrzyk, M. (2018). Application of statistical representation of the microstructure to modeling of phase transformations in DP steels by solution of the diffusion equation. Procedia Manufacturing, 15, 1847-1855.

Choudhury, A.N. (2013). Quantitative phase-field model for phase transformations in multi-component alloys. KIT Scientific Publishing.

Christian, J.W. (1975). The theory of transformations in metals and alloys. Pergamon Press.

Clouet, E., \& Soisson, F. (2010). Atomic simulations of diffusional phase transformations. Comptes Rendus Physique, 11(3-4), $226-235$.

Contieri, R.J., Zanotello, M., \& Caram, R. (2017). Simulation of CP-Ti recrystallization and grain growth by a cellular automata algorithm: simulated versus experimental results. Materials Research, 20(3), 688-701.

Das, S., Palmiere, E.J., \& Howard, I.C. (2002). CAFE: a tool for modelling thermomechanical processes. In E.J. Palmiere, M. Mahfouf, C. Pinna (Eds.), Conference proceedings. International Conference on Thermomechanical Processing: Mechanics, Microstructure \& Control. 23-26 June 2002, The University of Sheffield, England (pp. 296-301).

Donnay, B., Herman, J.C., Leroy, V., Lotter, U., \& Grossterlinden, R. (1996). Microstructure evolution of C-Mn Steels in the hot deformation process: The STRIPCAM model. In J.H. Beynon, P. Ingham, H. Teichert, K. Waterson, H. Pircher (Eds.), Proceedings of $2^{\text {nd }}$ Conference on Modelling of Metal Rolling Processes (pp. 23-35).

Estrin, Y., \& Mecking, H. (1984). A unified phenomenological description of work hardening and creep based on one-parameter model. Acta Metallurgica, 32(1), 57-70.

Fang, H., Mecozzi, M.G., Brück, E., Zwaag, S., van der, \& Dijk, N.H., van (2018). Analysis of the grain size evolution for ferrite formation in Fe-C-Mn steels using a 3D Model under a mixed-mode interface condition. Metallurgical and Materials Transactions A, 49(1), 41-53.

Fausty, J., Murgas, B., Florez, S., Bozzolo, N., \& Bernacki, M. (2020). A new analytical test case for the anisotropic grain growth problem - first calculations with a Finite Element Level Set model. Applied Mathematical Modelling, 93, $28-52$.

Fish, J. (Ed.) (2009). Multiscale methods: bridging the scales in science and engineering. Oxford University Press.

Fish, J. (2013). Practical multiscaling. Wiley.

Furstoss, J., Bernacki, M., Petit, C., Fausty, J., Muñoz, D.P., \& Ganino, C. (2020). Full field and mean field modeling of grain growth in a multiphase material under dry conditions: application to peridotites. JGR Solid Earth, 125(1), e2019JB018138.

Håkan, H. (2013). A modified level set approach to 2D modeling of dynamic recrystallization. Modelling and Simulation in Materials Science and Engineering, 21(8), 085012.

Halder, C., Madej, L., \& Pietrzyk, M. (2014). Discrete micro-scale cellular automata model for modelling phase transformation during heating of dual phase steels. Archives of Civil and Mechanical Engineering, 14(1), 96-103.

Janssens, K.G.F., Raabe, D., Kozeschnik, E., Miodownik, M.A., \& Nestler, B. (2007). Computational materials engineering: An introduction to microstructure evolution. Academic Press.

Johnson, W.A., \& Mehl, R.F. (1939). Reaction kinetics in processes of nucleation and growth. Transactions AIME, 135, 416-442. 
Karhausen, K., \& Kopp, R. (1992). Model for integrated process and microstructure simulation in hot forming. Steel Research, $63(6), 247-256$.

Kobayashi, S., Oh, S.I., \& Altan, T. (1989). Metal forming and the finite element method. Oxford University Press.

Колмогоров, А.Н. (1937). К статистической теории кристаллизации металлов. Известия Академии Наук СССР. Серия математическая, 1(3), 355-359.

Kuziak, R., Radwański, K., Molenda, R., Mazur, A., Broll, J., \& Pietrzyk, M. (2018). Identification of phase transformation model for the simulation of controlled cooling of bainitic steel tubes. Prace Instytutu Metalurgii Żelaza, 70, $24-33$.

Lan, Y.J., Li, D.Z., \& Li, Y.Y. (2004). Modeling austenite decomposition into ferrite at different cooling rate in low-carbon steel with cellular automaton method. Acta Materialia, 52(6), 1721-1729.

Le, C.T., Nguyen, Trang T., Nguyen, Thao T., \& Le, V.V. (2019). Molecular dynamics simulation of phase transformation and mechanical behavior in $\mathrm{Al}_{2} \mathrm{O}_{3}$ model. Vacuum, 167, 175-181.

Leblond, J.B., \& Devaux, J. (1984). A new kinetic model for anisothermal metallurgical transformations in steel including effect of austenite grain size. Acta Metallurgica, 32(1), 137-146.

Lian, Y., Lin, S., Yan, W., Liu, W.K., \& Wagner, G.J. (2018). A parallelized three-dimensional cellular automaton model for grain growth during additive manufacturing. Computational Mechanics, 61(5), 543-558.

Liu, E.Y., Zhang, D.H., Sun, J., Peng, L.G., Gao, B.H., \& Su L.T. (2012). Algorithm design and application of laminar cooling feedback control in hot strip mill. Journal of Iron and Steel Research, International, 19(4), 39-42.

Liu, X., Li, H., \& Zhan, M. (2018). A review on the modeling and simulations of solid-state diffusional phase transformations in metals and alloys. Manufacturing Review, 5, 10.

Madej, L. (2017). Digital/virtual microstructures in application to metals engineering - A review. Archives of Civil and Mechanical Engineering, 17(4), 839-854.

Madej, Ł., Mrozek, A., Kuś, W., Burczyński, T., \& Pietrzyk, M. (2008). Concurrent and upscaling methods in multi scale modelling - case studies. Computer Methods in Materials Science, 8(1), 1-10.

Maire, L. (2018). Full field and mean field modeling of dynamic and post-dynamic recrystallization in $3 D$-Application to $304 \mathrm{~L}$ steel ( $\mathrm{PhD}$ thesis). MINES ParisTech.

Marx, V., Reher, F.R., \& Gottstein, G. (1999). Simulation of primary recrystallization using a modified three-dimensional cellular automaton. Acta Materialia, 47(4), 1219-1230.

Mecking, H., \& Kocks, U.F. (1981). Kinetics of flow and strain-hardening. Acta Metallurgica, 29(11), 1865-1875.

Mecozzi, M.G., Militzer, M., Sietsma, J., \& Zwaag, S., van der (2008). The role of nucleation behavior in phase-field simulations of the austenite to ferrite transformation. Metallurgical and Materials Transactions A, 39(6), 1237-1247.

Mecozzi, M.G., Eiken, J., Santofimia, M.J., \& Sietsma, J. (2016). Phase field modelling of microstructural evolution during the quenching and partitioning treatment in low-alloy steels. Computational Materials Science, 112, part A, $245-256$.

Milenin, I., Pernach, M., \& Pietrzyk, M. (2015). Application of the control theory for modelling austenite-ferrite phase transformation in steels. Computer Methods in Materials Science, 15(2), 327-335.

Morkisz, P., Oprocha, P., Przybyłowicz, P., Czyzewska, N., Kusiak, J., Szeliga, D., Rauch, Ł., \& Pietrzyk, M. (2019). Prediction of distribution of microstructural parameters in metallic materials described by differential equations with recrystallization term. International Journal for Multiscale Computational Engineering, 17(3), 361-371.

Niño, O., Martínez, D.I., Lizcano, C., Guerrero-Mata, M., \& Colás, R. (2007). Study of the Tempcore process for the production of high resistance reinforcing rods. Materials Science Forum, 537-538, 533-540.

Osher, S., \& Sethian, J.A. (1988). Fronts propagating with curvature-dependent speed: Algorithms based on Hamilton-Jacobi formulations. Journal of Computational Physics, 79(1), 12-49.

Pereloma, E., \& Edmonds, D. (2012). Phase transformations in steels. Fundamentals and diffusion-controlled transformations ( $1^{\text {st }}$ ed., vol. 1). Woodhead Publishing.

Pietrzyk, M. (1990). Finite element based model of structure development in the hot rolling process. Steel Research, 61(12), 603-607.

Pietrzyk, M. (2000). Finite-element simulation of large plastic deformation. Journal of Materials Processing Technology, 106(1-3), 223-229.

Pietrzyk, M., \& Kuziak, R. (2012). Modelling phase transformations in steel. In J. Lin, D. Balint, M. Pietrzyk (Eds.), Microstructure evolution in metal forming processes (pp. 145-179). Woodhead Publishing.

Pietrzyk, M., \& Madej, L. (2017). Perceptive review of ferrous micro/macro material models for thermo-mechanical processing applications. Steel Research International, 88(10), 1700193.

Pietrzyk, M., Madej, L., Rauch, L., \& Szeliga, D. (2015). Computational Materials Engineering: Achieving high accuracy and efficiency in metals processing simulations. Elsevier.

Raabe, D. (2002). Cellular automata in materials science with particular reference to recrystallization simulation. Annual Review of Materials Research, 32, 53-76.

Raghavan, S., \& Sahay, S.S. (2007). Modeling the grain growth kinetics by cellular automaton. Materials Science and Engineering: A, 445-446, 203-209.

Roh, W., \& Kikuchi, N. (2002). Analysis of Stefan problem with level set method. In $8^{\text {th }}$ AIAA/ASME Joint Thermophysics and Heat Transfer Conference. American Institute of Aeronautics and Astronautics.

Rudnizki J., Böttger B., Prahl U., \& Bleck W. (2011). Phase-field modeling of austenite formation from a ferrite plus pearlite microstructure during annealing of cold-rolled dual-phase steel. Metallurgical and Materials Transactions A, 42(8), $2516-2525$.

Scheil, E. (1935). Anlaufzeit der Austenitumwandlung. Archiv für Eissenhüttenwesen, 8(12), 565-67. 
Schröder, J. (2014). A numerical two-scale homogenization scheme: the FE²-method. In J. Schröder, K. Hackl (Eds.), Plasticity and beyond. Microstructures, crystal-plasticity and phase transitions (pp. 1-64). Springer.

Schröder, J., Balzani, D., \& Brands, D. (2011). Approximation of random microstructures by periodic statistically similar representative volume elements based on lineal-path functions. Archives of Applied Mechanics, 81(7), 975-997.

Song, K.J., Wei, Y.H., Dong, Z.B., Wang, X.Y., Zheng, W.J., \& Fang, K. (2015). Cellular automaton modeling of diffusion, mixed and interface controlled phase transformation. Journal of Phase Equilibria and Diffusion, 36(2), 136-148.

Steinbach, I., \& Apel, M. (2006). Multi phase field model for solid state transformation with elastic strain. Physica D: Nonlinear Phenomena, 217(2), 153-160.

Strangwood, M. (2012). Chapter 6. Fundamentals of ferrite formation in steels. In E. Pereloma, D.V. Edmonds (Eds.), Phase transformations in steels (pp. 187-224). Woodhead Publishing.

Urcola, J.J., \& Sellars, C.M. (1987). Influence of changing strain rate on microstructure during hot deformation. Acta Metallurgica, 35(11), 2649-2657. 